\title{
The impact of HLA-ABDR mismatch on acute rejection and graft function among Filipino kidney transplant recipients
}

\author{
Mel- Hatra I. Arakama ${ }^{1}$, Glenda Eleanor P. Pamugas ${ }^{1}$ and Romina A. Danguilan ${ }^{\text {* }}$ \\ ${ }^{1}$ Department of Adult Nephrology, National Kidney and Transplant Institute, East Avenue, Quezon City, Philippines
}

\begin{abstract}
Aim: To determine the impact of HLA-A, B, DR mismatch on biopsy- proven acute rejection and renal graft function among renal transplant recipients with living and deceased donors from 1-3 years post-transplant.

Methods: Retrospective cohort study of recipients of kidney transplants performed between January 2008 - October 2011 at the National Kidney and Transplant Institute. They were classified according to donor source and the number of HLA-ABDR mismatch (0-6) and HLA-DR match (0-2)

Results: Nine hundred fifty-one (951) patients were included in the analysis. The rejection rate was highest in living donor kidney transplants with 6 HLA-ABDR $\mathrm{MM}$ who were not given induction (33.3\%). In living donor kidney transplants, the rejection rate was lower in all HLA-ABDR MM groups if induction therapy was given, even in the group with $6 \mathrm{HLA}-\mathrm{ABDR}$ MM. There was no statistically significant difference in the rate of biopsy proven acute rejection in the number of HLA-ABDR MM and HLA-DR MM. The use of eATG in LDKT and proliferative signal (PSI) inhibitors conferred a higher risk of acute rejection. No significant variation in estimated glomerular filtration rate (eGFR) was observed in living donor kidney transplants based on HLA-ABDR MM and HLA-DR MM at 1 to 3 years post-transplant. However, deceased donor kidney transplant patients had lower renal function on 3-year follow-up compared to those with living donors, and patients with 0 HLA-ABDR MM showed the best renal function at 3 years. The one year graft survival rate was $98.7 \%$ and the three year survival rate was $92.3 \%$.
\end{abstract}

Conclusion: There is no significant difference in acute rejection rate and renal graft function up to 3 years in living and deceased donor kidney transplants based on the number of HLA-ABDR and HLA-DR mismatch, using our current immunosuppressive protocols.

\section{Introduction}

Renal transplantation is the best possible mode of replacement therapy in most patients with chronic kidney disease. In the Philippines, kidney transplantation has improved considerably in both graft and patient survival. It offers a superior prognosis $[1,2]$ and an improved quality of life [3] and is more cost-effective compared to any form of dialysis. Advances have been made in tissue typing technology, immunosuppression, the diagnosis and treatment of acute and chronic allograft dysfunction, and the diagnosis and treatment of immunosuppression-related problems [4,5].

There are many factors that correlate with graft survival. Human leukocyte antigens (HLA) play a vital part in the cellular and humoral immune responses that determine the outcome of a transplant. The role of HLA matching in renal transplantation is changing with improvements in the understanding of the structure and function of HLA and as advances in technology have improved our capability to differentiate HLA antigens and the antibodies reactive to them. Transplant success rates have markedly increased because of the improvement in immunosuppression strategies. The benefit of HLA matching varies depending on donor and recipient risk factors [7].

Local studies showed that the graft survival rate of kidney transplants done from January 2002 to December 2007 were $89.9 \%$, $89.9 \%$ and $83.4 \%$ at 1 year, 3 years and 5 years respectively, for deceased donor [8]. On the other hand, the graft survival rate of living donor kidney transplants done from January 1986 to December 1996 were $84.77 \%, 74.63 \%$ and $50.61 \%$ at 1 year, 2 years and 5 years, respectively [9].

Before 1985, the 1-year graft loss for HLA-matched grafts in the United States was $17 \%$ compared with $42 \%$ for those with 6 ABDR mismatched (MM) antigens (a 25\% difference) [8]. In 1990, this difference decreased to $17 \%$ [10]. In 1995, the rate of graft loss was $10 \%$ for HLA matched and $18 \%$ for HLA-mismatched transplants [11]. In 2001, only $7 \%$ of HLA-matched and $12 \%$ of HLA-mismatched transplants were lost-a fourfold decrease from 1985 [12]. The potent immunosuppressive protocols suppressed early graft loss from rejection $[11,12]$.

\section{Significance of the study}

Protocols on the use of immunosuppressive medications have been tailored to the immunologic risk of the recipient, based on the number of HLA mismatch and level of the recipient's sensitization. In spite of

Correspondence to: Romina A. Danguilan, Department of Adult Nephrology, National Kidney and Transplant Institute, East Avenue, Quezon City, Philippines, E-mail: dr.radanguilan@gmail.com

Key words: living donor kidney transplant, deceased donor kidney transplant, HLA-ABDR mismatch, biopsy-proven acute rejection, induction therapy

Received: April 02, 2017; Accepted: April 26, 2017; Published: April 29, 2017 
the excellent graft survival rates, there is still some apprehension about performing kidney transplants for 6 HLA mismatched pairs. HLA DR mismatch is considered an important factor in allocation of a deceased donor kidney to a recipient on the waiting list and in the type and degree of immunosuppression given [13].

\section{General objective:}

1. To determine the impact of HLA ABDR mismatch (MM) on biopsy- proven acute rejection and renal graft function among renal transplant recipients with living and deceased donors

\section{Specific objectives:}

1. To correlate the number of HLA ABDR and HLA DR mismatch with the incidence of biopsy proven acute rejection

2. To determine renal graft function at one to three years post transplant according to HLA ABDR and HLA DR mismatch

3. To determine the association of induction therapy and oral immunosuppression with the incidence of biopsy- proven acute rejection and renal graft function

\section{Methodology}

This was a retrospective cohort study in a tertiary hospital setting which reviewed all adult recipients who underwent kidney transplantation from living and deceased donors between January 2008 to October 30, 2011 at the National Kidney and Transplant Institute (NKTI).

\section{Inclusion Criteria:}

1. Filipino renal transplant recipients $>18$ years old

2. With living or deceased kidney donor

3. Panel Reactive Antibody (PRA) $<30 \%$

\section{Exclusion Criteria:}

1. History of positive crossmatch

2. Presence of Donor Specific Antibodies (DSA)

3. Underwent plasmapheresis

4. Patients who were lost to follow up

\section{Data collection}

The study sources included hospital records and the Philippine Renal Disease Registry/Renal Disease Control Program (REDCOP) database. The following demographic data were obtained: age, sex, number of total HLA ABDR mismatch and HLA-DR mismatch, age at transplantation, cause of end-stage renal disease, duration of pre transplantation dialysis, donor source (deceased or living), pretransplantation panel reactive antibody (PRA) level, induction therapy and immunosuppression used. Outcome measures included the occurrence of biopsy-proven acute cellular or humoral rejection and renal function at 1-3 years post transplant.

\section{Data analysis}

A chi-square based statistical analysis was used to test whether distributions of categorical variables differed from one another. Logistic regression showed the odds ratio in each category of total HLA mismatch and DR mismatch over the reference 0 mismatch. Rejection rates per number of HLA mismatch as well as the mean distribution of renal graft function using CKD Epi equation were obtained.

\section{Results}

We reviewed the data of 1072 recipients of kidney transplant performed between January 2008- October 2011 in our Institution. We excluded 32 foreign transplant recipients, 15 highly sensitized patients, and 74 patients who were lost to follow-up. Patients were classified according to donor source (living or deceased), number of total HLA- ABDR mismatch (0-6) and DR mismatch (0-2). There were 951 patients were included in the analysis (Table 1 ).

There were 805 living donor kidney transplants (LDKT) and 156 deceased donor kidney transplants (DDKT). Most of the recipients were between the ages of 31- 45 years old (37.89\%) among the living donor kidney transplants while $47 \%$ of the deceased donor kidney transplant recipients were in the age range of 46- 60 years old. Most of the kidney transplant recipients were females and were on dialysis for less than a year. The PRA Class I was less than $10 \%$ in all patients and PRA class II was less than $10 \%$ in the majority of patients. Chronic glomerulonephritis (CGN) (53.91\%) was the most common native kidney disease among recipients with living donors while diabetes mellitus and CGN were the most common among recipients with deceased donors. Majority of the patients was given induction therapy with Basiliximab (38.4\%) and had Tacrolimus-based immunosuppression (58.26\%). Almost half of the living donor kidney transplants had 3 and 4 HLA ABDR mismatch (49.44\%) and 1 DR mismatch (49\%). Most of the recipients with deceased donors had 4 HLA ABDR mismatch (26.71\%). Overall, only 55 patients had 0 ABDR MM with their donor and less than $5 \%$ had 6 ABDR mismatch.

\section{Acute rejection}

Overall, the rejection rate was highest in LDKT with 6 HLA MM without induction (33.3\%), in DDKT with 0 HLA MM not given induction (20\%) and with 4 HLA MM with induction (18\%). (Table II)

Among LDKT, no rejection episode was seen in 0 HLA MM with induction therapy. Except in the 5 HLA MM group, the rejection rate was higher if no induction therapy was given. The rejection rate in LDKT 6 HLA MM group with induction therapy (9.4\%) was lower than in LDKT with 2 HLA MM up to 5 HLA MM with no induction therapy. Among recipients who were not given induction therapy, the highest rejection rate was in 6HLA MM (33.3\%) and lowest in the 0 HLA MM (8\%). Among patients who received induction therapy, the rejection rate was highest in 4 HLA MM (12.1\%) and there were no rejection episodes in recipients who had 0 HLA MM.

For patients with deceased donors (DDKT) almost all received induction therapy except for 5 patients with 0 HLA DR MM. No rejection episode was seen in the 0 HLA MM group with induction and in the 6 HLA MM group, who all received induction therapy. The highest rate of rejection was seen in patients with $4 \mathrm{MM}(18 \%)$.

The acute rejection rate was highest among LDKT recipients with 2 HLA DR MM (15.2\%) and who did not receive induction therapy. Patients who received induction therapy had lower rejection rates compared to those without induction across all three groups.

For DDKT recipients, 1 out of 4 patients with 0 HLA DR MM and who did not receive induction therapy had acute rejection (20\%). For recipients who received induction, the rejection rate was lowest in the 2 HLA DR MM group (6.97\%) and highest in the 0 HLA DR MM group $(14.8 \%)$.

The odds ratio for each number of HLA ABDR mismatch over the reference 0 HLA ABDR mismatch for both living donor and deceased 
Table 1. General characteristics of kidney transplant patients from January 2008- October 2011 by donor source (N=951).

\begin{tabular}{|c|c|c|c|c|}
\hline & \multicolumn{2}{|c|}{$\begin{array}{l}\text { Living Donor Kidney Transplant } \\
\qquad(\mathrm{N}=805)\end{array}$} & \multicolumn{2}{|c|}{$\begin{array}{l}\text { Deceased Donor Kidney Transplant } \\
\qquad(N=146)\end{array}$} \\
\hline Age of Recipient (years) & \multicolumn{2}{|c|}{$\mathrm{N}(\%)$} & \multicolumn{2}{|c|}{$\mathrm{N}(\%)$} \\
\hline $18-30$ & 190 & $23.60 \%$ & 18 & $12.33 \%$ \\
\hline $31-45$ & 305 & $37.89 \%$ & 42 & $28.77 \%$ \\
\hline $46-60$ & 229 & $28.45 \%$ & 61 & $41.78 \%$ \\
\hline$>60$ & 81 & $10.06 \%$ & 25 & $17.12 \%$ \\
\hline \multicolumn{5}{|c|}{ Sex of Recipient } \\
\hline Female & 528 & $65.59 \%$ & 94 & $64.38 \%$ \\
\hline Male & 277 & $34.41 \%$ & 52 & $35.62 \%$ \\
\hline \multicolumn{5}{|c|}{ Native Kidney Disease } \\
\hline Diabetes & 149 & $18.51 \%$ & 50 & $34.25 \%$ \\
\hline HPN & 147 & $18.26 \%$ & 36 & $24.66 \%$ \\
\hline Glomerulonephritis & 434 & $53.91 \%$ & 50 & $34.25 \%$ \\
\hline Obstuctive Uropathy & 18 & $2.24 \%$ & 3 & $2.05 \%$ \\
\hline Autosomal Polycystic Kidney Disease & 9 & $1.12 \%$ & 0 & $0.00 \%$ \\
\hline Chronic Pyelonephritis & 48 & $5.96 \%$ & 7 & $4.79 \%$ \\
\hline \multicolumn{5}{|c|}{ Years on Dialysis Before Transplant } \\
\hline None & 86 & $10.68 \%$ & 17 & $11.64 \%$ \\
\hline$<12$ months & 457 & $56.77 \%$ & 61 & $41.78 \%$ \\
\hline 12-24months & 171 & $21.24 \%$ & 40 & $27.40 \%$ \\
\hline$>24$ months & 91 & $11.30 \%$ & 28 & $19.18 \%$ \\
\hline \multicolumn{5}{|c|}{ Total HLA ABDR Mismatch } \\
\hline 0 & 48 & $5.96 \%$ & 7 & $4.79 \%$ \\
\hline 1 & 88 & $10.93 \%$ & 12 & $8.22 \%$ \\
\hline 2 & 136 & $16.89 \%$ & 18 & $12.33 \%$ \\
\hline 3 & 193 & $23.98 \%$ & 34 & $23.29 \%$ \\
\hline 4 & 186 & $23.11 \%$ & 39 & $26.71 \%$ \\
\hline 5 & 119 & $14.78 \%$ & 25 & $17.12 \%$ \\
\hline 6 & 35 & $4.35 \%$ & 11 & $7.53 \%$ \\
\hline HLA DR Mismatch & Total & $\%$ & & \\
\hline 0 & 219 & $27.20 \%$ & 32 & $21.92 \%$ \\
\hline 1 & 398 & $49.44 \%$ & 71 & $48.63 \%$ \\
\hline 2 & 188 & $23.35 \%$ & 43 & $29.45 \%$ \\
\hline \multicolumn{5}{|c|}{ Panel- Reactive Antibodies } \\
\hline \multicolumn{5}{|l|}{ Class I } \\
\hline $0-10 \%$ & 805 & $100.00 \%$ & 146 & $100.00 \%$ \\
\hline $11-30 \%$ & 0 & $0.00 \%$ & 0 & $0.00 \%$ \\
\hline \multicolumn{5}{|l|}{ Class II } \\
\hline $0-10$ & 524 & $65.09 \%$ & 92 & $63.01 \%$ \\
\hline $11-30$ & 277 & $34.41 \%$ & 53 & $36.30 \%$ \\
\hline \multicolumn{5}{|c|}{ Induction } \\
\hline None & 227 & $28.20 \%$ & 5 & $2.7 \%$ \\
\hline rATG & 110 & $13.66 \%$ & 43 & $29.4 \%$ \\
\hline Alemtuzumab & 104 & $12.92 \%$ & 15 & $10.3 \%$ \\
\hline Basiliximab & 265 & $32.92 \%$ & 56 & $38.4 \%$ \\
\hline Daclizumab & 60 & $7.45 \%$ & 9 & $6.2 \%$ \\
\hline Rituximab & 10 & $1.24 \%$ & 0 & $0.00 \%$ \\
\hline eATG & 29 & $3.60 \%$ & 18 & $6.8 \%$ \\
\hline \multicolumn{5}{|c|}{ Immunosuppression } \\
\hline Tacrolimus, Mycophenolate, Prednisone & 469 & $58.26 \%$ & 90 & $61.64 \%$ \\
\hline CYA, Mycophenolate, Prednisone & 319 & $39.63 \%$ & 54 & $36.99 \%$ \\
\hline Proliferative Signal Inhibitor & 17 & $2.11 \%$ & 2 & $1.37 \%$ \\
\hline
\end{tabular}

donor kidney transplants. Logistic regression analysis showed no statistically significant difference in acute rejection in patients with 1 HLA ABDR MM up to 6 HLA ABDR MM against 0 HLA ABDR MM.

Likewise, the odds ratio of 1 HLA DR MM and 2 HLA DR MM were not statistically significant compared to zero HLA DR mismatch, indicating that there is no statistical difference in biopsy proven acute rejection rates in the three groups for both living and deceased donor kidney transplants. To evaluate if there was a difference in acute rejection rates if induction or no induction was given and the type of induction treatment administered, a logistic regression analysis was performed. For both the living and deceased donor kidney transplant recipients, use of eATG had the highest likelihood of rejection compared to no 
induction. There was no statistically significant difference in acute rejection rate for all other types of induction treatment compared to no induction.

Among LDKT recipients who received tacrolimus-based immunosuppression, the highest rejection rate occurred in patients who had 4 HLA MM (13.3\%), with no rejection in recipients who had 1 HLA MM. For recipients who were on cyclosporine (CYA)- based immunosuppression, rejection rate was highest among patients who had 6 HLA MM (14.3\%) and lowest among recipients who had 0 HLA MM (3.4\%). Only 17 patients were given PSI as initial immunosuppression.

In DDKT recipients, rejection rates were lower among those on tacrolimus- based immunosuppression compared to CYA, except in the 3 HLA MM group were none of the 9 patients developed rejection.

The odds ratio of rejection rate by initial immunosuppression with Tacrolimus-based regimen as reference category. There is no statistical difference in the rejection rate between CYA- and PSIbased maintenance immunosuppression in LDKT. But in DDKT, the odds ratio of CYA- based and PSI- based immunosuppression was significantly different compared to Tacrolimus. The probability of rejection with CYA is 4.886 times higher and 21.5 times higher with PSI compared to Tacrolimus- based immunosuppression.

\section{Renal function}

Renal graft function using estimated glomerular function rate (eGFR) by CKD-Epi formula at 1,2 and 3 years post-transplant was also determined in this study.

Overall, there was no significant variation in the mean eGFR of living donor kidney transplant from 1-3 years post transplant. Among deceased donor kidney transplants, mean eGFR increased to $74.85 \mathrm{ml} /$ $\mathrm{min}$ in the second year post-transplant but was comparable at 1 and 3 years.

At one-year post transplant, most of the LDKT recipients had eGFR of $>60 \mathrm{ml} / \mathrm{min}$ for all HLA ABDR MM groups (73\% in $0 \mathrm{MM}, 77 \%$ in $6 \mathrm{MM}$ ). Patients with eGFR less than $30 \mathrm{ml} / \mathrm{min}$ comprised only $3-6 \%$ in all groups. In the 0 HLA ABDR MM group, there was an increase in the proportion of patients with eGFR $>60 \mathrm{ml} / \mathrm{min}$ from $79 \%$ at one year to $83 \%$ at 3 years. In the three-year period, no significant variation was seen in renal function in the different groups.

Likewise, there was no significant variation in the eGFR at 1-3 years in patients with 0,1 and 2 HLA-DR mismatch, where the proportion of patients with eGFR $>60 \mathrm{ml} / \mathrm{min}$ was maintained at $>70 \%$.

Likewise, there was no significant variation in the eGFR at 1-3 years in patients with 0,1 and 2 HLA-DR mismatch, where the proportion of patients with eGFR $>60 \mathrm{ml} / \mathrm{min}$ was maintained at $>70 \%$.

Among deceased donor kidney transplants, the proportion of patients with eGFR $>60 \mathrm{ml} / \mathrm{min}$ was highest among patients with 0 HLA MM and 6 HLA MM compared to other groups at one year. Half of patients with 3 HLA MM had eGFR of 30-60 $\mathrm{ml} / \mathrm{min}$. Patients with deceased donors had a higher proportion of eGFR $<60 \mathrm{ml} / \mathrm{min}$ at 1 year for 1- 5 HLA ABDR MM groups compared to those with living donors.

Likewise, the proportion of patients with eGFR $<30 \mathrm{ml} / \mathrm{min}$ was higher in all HLA MM groups with deceased donors compared to recipient with living donors with the same number of mismatch.

In recipients with 0 HLA MM and 1 HLA MM, the proportion of patients with eGFR $<30 \mathrm{ml} / \mathrm{min}$ decreased from $14 \%$ and $17 \%$ to zero. Patients with 0 HLA MM had the best renal function, with $83 \%$ having eGFR $>60 \mathrm{ml} / \mathrm{min}, 17 \%$ with GFR between $30-60 \mathrm{ml} / \mathrm{min}$ and no patients with eGFR $<30 \mathrm{ml} / \mathrm{min}$. The graft survival rate at 1 year of was $98.7 \%$.

\section{Discussion}

Analysis of effect of number of HLA mismatch on biopsy proven rejection in relation to induction therapy and initial oral immunosuppression showed that there is no statistically significant difference between the number of HLA-ABDR MM and the incidence of biopsy-proven acute rejection in both living and deceased donor kidney transplants. This can be attributed to the increasing use of induction therapy and the tailoring of immunosuppressive medications based on the HLA mismatch, with more potent medications being given to patients with a greater number of mismatch and even for 0 mismatch. Indeed, data from the Philippine Renal Disease Registry shows that from 2009-2011, 77-81\% of patients received induction therapy, regardless of the number of mismatch. In general, almost all patients with deceased donors received induction therapy, and the highest rejection rate (20\%) was shown in the group with $0 \mathrm{MM}$ and who did not receive induction. Globally, the standard immunosuppressive protocol is tacrolimus-based. No significant difference in rejection rates was seen between patients on tacrolimus-based triple drug regimens or cyclosporine-based regimens.

The highest rejection rates were observed with the use of equine ATG (eATG), which is no longer used in the institution for induction and proliferative signal inhibitors (PSIs,) which are rarely used as a de novo immunosuppression agent.

A limitation of the study is that only the initial immunosuppressive drug was studied, as we could not determine if there was a shift to another immunosuppressive drug during the study period. We also could not account for the difference in the doses and target blood levels of the drug, and the patient's compliance in taking his maintenance medication because of the retrospective study design. A shift to another oral immunosuppressant is possible due to rejection, infection or drugrelated adverse effects. Another limitation of this study is that we did not account for death of the patient or return to dialysis after graft loss, which could be reasons why patients were lost to follow-up.

Renal function based on eGFR in living donor kidney transplants did not change significantly during the 3-year follow-up, regardless of HLA mismatch. Even with our success in preventing acute rejection in deceased donor kidney transplants, long-term renal function is better in living donor kidney transplants over the study period. With deceased donor kidney transplants 0 HLA-ABDR MM patients had the lowest proportion of eGFR $<30 \mathrm{ml} / \mathrm{min}$ at 3 years. Immunologic factors, as well as factors inherent to the donor kidney, such as length of the cold ischemia time during harvest, age of the donor, and co-morbid medical conditions such as hypertension, diabetes and arteriosclerosis, contribute to graft survival long-term. In general, living donors are screened more meticulously pre-transplant, and disqualified immediately once with medical contraindications because of concerns regarding complications for the donor over time [14-20].

\section{Conclusions and recommendations}

There is no significant difference in acute rejection rate and renal graft function up to 3 years in living donor and deceased donor kidney transplants based on the number of HLA-ABDR and HLA-DR mismatch, using our current immunosuppressive protocols. Kidney transplantation with medically suitable living donors, regardless of the 
number of HLA-ABDR mismatch, shows better long-term outcomes than for deceased donors. A prospective study with longer follow-up period is recommended for better evaluation of factors that affect graft and patient survival [20-22].

\section{References}

1. Kanwar M, Hashem M, Rosman H, Kamalakannan D, Cheema A, et al. (2006) Usefulness of clinical evaluation, troponins, and C-reactive protein in predicting mortality among stable hemodialysis patients. Am J Cardiol 98: 1283-1287. [Crossref]

2. Apple FS, Murakami MM, Pearce LA, Herzog CA (2004) Multi-biomarker risk stratification of $\mathrm{N}$-terminal pro-B-type natriuretic peptide, high-sensitivity $\mathrm{C}$-reactive protein, and cardiac troponin $\mathrm{T}$ and $\mathrm{I}$ in end-stage renal disease for all-cause death. Clin Chem 50: 2279-2285. [Crossref]

3. Niu SF, Li IC (2005) Quality of life of patients having renal replacement therapy. $J A d v$ Nurs 51: 15-21. [Crossref]

4. Øien CM, Reisaeter AV, Leivestad T, Dekker FW, Line PD, et al. (2007) Living donor kidney transplantation: the effects of donor age and gender on short- and long-term outcomes. Transplantation 83: 600-606. [Crossref]

5. Drognitz O, Donauer J, Kamgang J, Baier P, Neeff H, et al. (2007) Living-donor kidney transplantation: the Freiburg experience. Langenbecks Arch Surg 392: 23-33. [Crossref]

6. Takemoto SK, Terasaki PI, Gjertson DW, Cecka JM (2000) Twelve years' experience with national sharing of HLA-matched cadaveric kidneys for transplantation. $N$ Engl $J$ Med 343: 1078-1084. [Crossref]

7. www.ustransplant.org

8. Terasaki PI (2000) The HLA-matching effect in different cohorts of kidney transplant recipients. Clin Transpl. [Crossref]

9. Mallari, Jd., M.D., Sagayaga, HM., M.D, Danguilan, RA et. al, M.D. Graft and Recipient Outcome of the Deceased Donor Renal Transplantation Program at the National Kidney and Transplant Institute from 2002 - 2007; NKTI Proceedings.

10. Sancho- Ampil, R, M.D., Liquete,RM, M.D, Danguilan, RA, M. D. Graft Survival Analysis of Kidney transplant Patients from Living donors: The NKTI Experience.
11. Muczynski KA, Ekle DM, Coder DM, Anderson SK (2003) Normal human kidney HLA-DR-expressing renal microvascular endothelial cells: characterization, isolation, and regulation of MHC class II expression. J Am Soc Nephrol 14: 1336-1348.

12. Lewis RM, Janney RP, Golden DL (1989) Stability of renal allograft function associated with long-term cyclosporine immunosuppressive therapy-Five-year followup. Transplantation 47: 266-272.

13. Doxiadis II, de Fijter JW, Mallat MJ, Haasnoot GW, Ringers J, et al. (2007) Simpler and equitable allocation of kidneys from postmortem donors primarily based on full HLADR compatibility. Transplantation 83: 1207-1213.

14. Takiff H, Cook DJ, Himaya NS, Mickey MR, Terasaki PI (1988) Dominant effect of histocompatibility on ten-year kidney transplant survival. Transplantation 45: 410-415. [Crossref]

15. Øien CM, Reisaeter AV, Leivestad T, Dekker FW, Line PD, et al. (2007) Living donor kidney transplantation: the effects of donor age and gender on short- and long-term outcomes. Transplantation 83: 600-606. [Crossref]

16. Takemoto S, Carnahan E, Terasaki PI (1992) Beneficial effect of sharing six-antigenmatched cadaver kidneys in the United States. Transplant Proc 24: 1310-1311. [Crossref]

17. Gilks WR, Bradley BA, Gore SM, Klouda PT (1987) Substantial benefits of tissue matching in renal transplantation. Transplantation 43: 669.

18. Wynn JJ, Pfaff WW, Patton PR, Peterson JC, Salomon DR, et al. (1988) Late results of renal transplantation. Transplantation 45: 329-333. [Crossref]

19. Takemoto SK, Terasaki PI, Gjertson DW, Cecka JM (2000) Twelve years' experience with national sharing of HLA-matched cadaveric kidneys for transplantation. $N$ Engl $J$ Med 343: 1078-1084. [Crossref]

20. Stegall MD, Dean PG, McBride MA, Wynn JJ; Organ Procurement and Transplantation Network/United Network for Organ Sharing Kidney/Pancreas Transplantation Committee (2002) Survival of mandatorily shared cadaveric kidneys and their paybacks in the zero mismatch era. Transplantation 74: 670-675. [Crossref]

21. Doxiadis II, Rahmel A, Claas FH (2010) Towards kidney allocation on basis of HLADR compatibility. Clin Transpl. [Crossref]

22. Foss A, Leivestad T, Brekke IB, Fauchald P, Bentdal O, et al. (1998) Unrelated living donors in 141 kidney transplantations: a one-center study. Transplantation 66: 49-52. [Crossref]

Copyright: (C2017 Arakama MHI. This is an open-access article distributed under the terms of the Creative Commons Attribution License, which permits unrestricted use, distribution, and reproduction in any medium, provided the original author and source are credited. 